\title{
GENERATION OF PLASMA FLOWS AND FILAMENTATION OF MAGNETIC FIELDS IN THE SOLAR ATMOSPHERE
}

\author{
MARGARITA RYUTOVA \\ Institute of Nuclear Physics, 630090 Novosibirsk, 90, Russia
}

\begin{abstract}
Nonlinear effects in dynamics of solar magnetic fields which, in particular, determine the evolution of solar magnetic structures and their lifetimes, are discussed. By some analogy with the effects of acoustic streaming in usual hydrodynamics the general definition of these effects as "magneto acoustic streaming" is proposed.
\end{abstract}

The wonderful effects of acoustic streaming appeared to have an analogy in MHD (Ryutova 1986).

The origin of acoustic streaming, often called a "quartz wind", is connected with the presence of solid obstacles or solid boundary walls in a field of a sound wave: near the obstacle sound waves (or other kinds of oscillations) result in an additional tangential tension which provides the absorption of the energy of oscillations and leads to the generation of secondary flows in a thin "acoustic boundary layer".

After Faraday's (1831) short report on the upward and downward mass flows observed above the vibrating membrane, and the first analytical description of secondary mass flows by Lord Rayleigh (1884), the various manifestation of the effects of acoustic streaming in hydro- and gas-dynamics were found (see, for example, Nyborg, 1965 ).

These effects seem to be especially rich in magneto hydrodynamics. Even the very first steps (Ryutova, 1986) show not only the existence of these effects in plasma containing the structured magnetic fields, but also new effects which are absent in classical hydro- and gas-dynamics.

1. Unlike the solid obstacles, solar magnetic flux tube (or any other magnetic structure) is the flexible "body" surrounded by plasma. Now, the additional tensions appear both outside and inside the boundary of an oscillating flux tube. So that, besides the "acoustic boundary layer" outside a magnetic flux tube there is a "magneto acoustic boundary layer" inside it. Secondary flows are generated both outside and inside a flux tube. Of these two flows the inner streaming one plays the most important role for flux tube dynamics: "magneto acoustic streaming" drags the magnetic field and distorts it; the specific distortion depends on the physical parameters of the flux tube and its environment. 
2. There are two different mechanisms leading to magneto acoustic streaming (Ryutova, 1986): 1) the action of ponderomotive forces; and, 2) the absorption of the momentum and angular momentum of flux tube oscillations. The absorption of angular momentum causes the rotational mass flow across the flux tube axis, and absorption of momentum leads to upward (or downward) mass flows.

3. If the absorption is provided mostly with one of the plasma component (electron or ion, the specific situation depends on the damping mechanism), magneto acoustic streaming is accompanied by current drive. The geometry of currents corresponds to that of generated plasma flows. Generated currents change the equilibrium conditions of magnetic fields.

4. Specifics of solar magnetic flux tubes a priori provide the generation of magneto sonic streaming: even in the absence of any dissipative effects flux tube oscillations are damped away due to the action of two specific mechanisms. These are radiative damping of flux tube oscillations (Ryutov and Ryutova, 1976), and the absorption of oscillations in an Alfven resonance layer (Ryutova, 1977).

5. There are two principal regimes for the development of magneto acoustic streaming depending on the physical parameters of magnetic flux tube and its surroundings. One regime corresponds to the generation of stochastic motions which lead to the gradual (and diffusive) dissolving of flux tubes in the ambient plasma (this case occurs preferably for smallest scale magnetic flux tubes). Another regime is realized due to the regular magneto acoustic streaming leading to the clear filamentation of flux tubes: the initial magnetic flux tube breaks down into a complex structure with many magnetic elements of different strengths and sizes. The process of filamentation goes on until the new-born flux tubes reach the regime of diffusive dissolving. Thus, the lifetime of a particular magnetic flux tube is determined by its evolution: either a flux tube disappears diffusively never reaching the regime of filamentation, or it lives as long as the filamentation process is going on, and then starts to disappear. Outer new-born flux tubes disappear earlier than the inner ones.

6. In stratified atmosphere the split magnetic flux tube will branch off, and, since new-born flux tubes should not at all be identical (cf. our results of computer simulation, Figs.1-2), they will start to diverge at different heights, leading to the formation of a "magnetic tree".

The effects described in points 1-6 are quite general and can occur in other astrophysical objects containing structured magnetic fields and able to sustain any kind of acoustic or MHD oscillations.

The general approach to the analytical problem is as follows:

1. The linearized MHD-equations describing the interaction of magnetic flux tubes with the acoustic wave train (Ryutov and Ryutova, 1976;) give the first order solution for the flux tube perturbation amplitudes of flux tubes.

2. The solution of linearized equations determines the second order perturbations. The equation for the stationary velocity field of generated flows $V$ has a form:

$$
\rho \nu \nabla^{2} V=-\nabla\left(p+\frac{B^{2}}{4 \pi}\right)+F
$$


where $\nu$ is kinematic viscosity and $F$ is either a ponderomotive force acting on a unit volume of plasma, or the force connected with the specific damping mechanism of flux tube oscillations:

$$
F=-<\tilde{v} \operatorname{div} \rho \tilde{v}+\rho(\tilde{v} \nabla) \tilde{v}>+\frac{1}{4 \pi}<[\operatorname{rot} \tilde{B}, \tilde{B}]>.
$$

Tilde marks the linear perturbations.

It is obvious that secondary streaming can arise only if

$$
\operatorname{rot} F \neq 0 \text {. }
$$

Otherwise, the action of $F$ leads only to insignificant redistribution of plasma parameters inside the flux tube.

The field of forces determines the geometry of the generated velocity field, and results in a change of the magnetic field topology: plasma density and magnetic field become constant along the force field lines.

The evolution of the magnetic field depends on the relation between the the duration of the initial acoustic wave train, $T$, and the viscous time $\tau_{\nu} \approx R^{2} / \nu$ ( $R$ is the flux tube radius).

In the case of a coherent wave train, when $T>\tau_{\nu}$, the plasma density and magnetic field which follow the ponderomotive force lines have enough time for reorganization which ends up by "splitting" a flux tube into four independent tubes.

If the same conditions between $T, \tau_{\nu}$, and $R$ remain valid for new-born tubes, each of them experiences a further splitting.

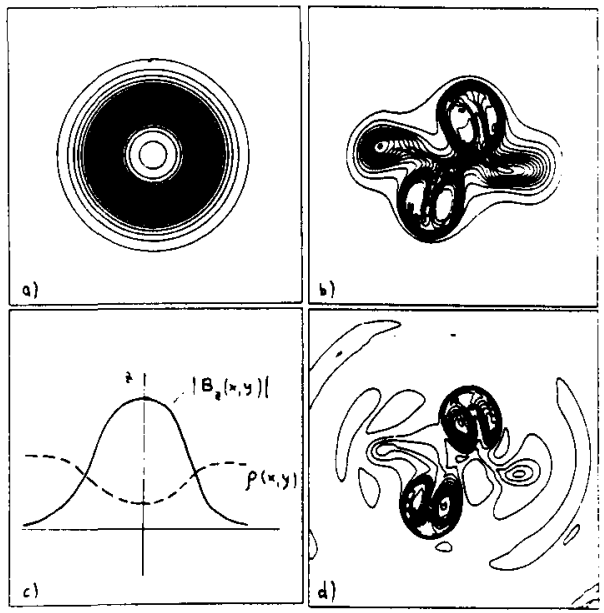

Fig. 1. The case when the $x$-component of the interaction force is much larger than y-component. Shown are: (a) initial $(t=0)$ contour lines of magnetic field $B$, and (c) the corresponding model of unperturbed cylindrical flux tube; final states of magnetic field (b) and density distribution $(\log \rho)(\mathrm{c})$. 
The process of filamentation goes on until the amplitude of generated flows $|V|$ becomes too small, and the condition $T<R /|V|$ becomes valid. Under this condition another regime of the flux evolution starts: the plasma density and magnetic field are smoothing out in a diffusive way. The inverse "diffusion coefficient" determines the time interval during which the flux tube is dissolving in the ambient plasma.

A computer simulation of the problem connected with the action of ponderomotive force was recently performed using the whole set of $2 \mathrm{D}$ nonlinear MHD equations with the right hand sides containing the interaction terms between flux tube and incident acoustic waves (Ryutova, Kaisig, and Tajima, 1992). Results for two different regimes are shown in Figs.1 and 2.

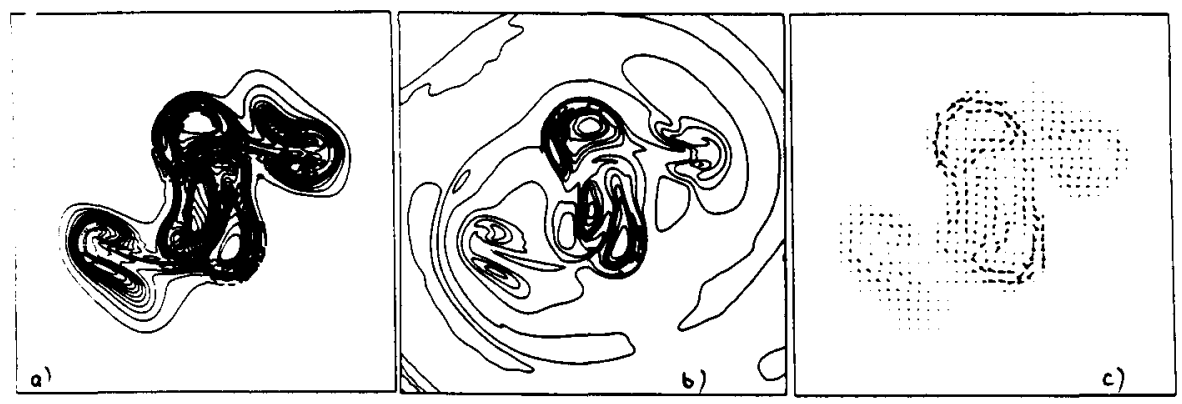

Fig. 2. The final state of (a) contour lines of $B,(\mathrm{~b})$ density contours $(\log \rho)$, and (c) density of generated currents $j=\left(j_{x}, j_{y}\right)$ for the case when both components of interaction force are of the same order: the intial flux tube (Fig.1c) breaks down into a complex structure with many magnetic elements.

In the case of incoherent wave train, when $T<\tau_{\nu}$, the generated flows have a character of stochastic motions from the very beginning. These motions result in a diffusive broadening of a flux tube which ends up in a complete dissolving of the magnetic field in the ambient plasma. The "diffusion coefficient" corresponding to this process is $D \sim T^{3} \xi^{4} \omega^{2} / R^{2}$, where $\xi$ is the displacement amplitude in linear oscillations of the flux tube. The lifetime of flux tube can be estimated as

$$
t_{D} \sim R^{4} / T^{3} \xi^{4} \omega^{2}
$$

Other mechanism of generation of magnetosonic streaming is similar to those in usual hydrodynamics and is connected with the absorption of the energy of oscillation. As it was mentioned above, this mechanism works for magnetic flux tubes a priori even in the absence of usual dissipative effects and is provided by: 1) the radiative damping of flux tube oscillations (Ryutov 
and Ryutova, 1976), and, 2) by the anomalous damping in an Alfven resonance layer (Ryutova, 1977).

Formally, the expression for the force has the same form as Eq.(2), but

now the terms which are directly connected with the absorption mechanism are taken into account.

The absorption of angular momentum causes rotational mass flow across the flux tube axis, and absorption of momentum leads to upward (or downward) mass flows.

If the absorption is provided mostly with one of the plasma components (electron or ion, the specific situation depends on the damping mechanism), magneto acoustic streaming is accompanied by current drive. The geometry of currents corresponds to that of generated plasma flows (cf. Fig.2 c).

\section{REFERENCES}

Faraday M., 1831, Phil. Trans. Roy. Soc. London, 121, 229.

Nyborg W.L., 1965, in Physical Acoustics, Vol. 2, Part B, Properties of Polimers and Nonlinear Acoustics, Academic Press.

Rayleigh, Lord, 1884, Phil. Trans. Roy. Soc. London, 175, 1.

Ryutov D.D., Ryutova M.P., 1976, Sov. Phys. JETP, 43, 491..

Ryutova M.P., 1977, XIII Int. Conf. on Phenomena in Ionized Gases, Berlin, p. 859.

Ryutova M.P., 1986, Proc. of Joint Varenna-Abastumani Int. School on Plasma Astrophysics, ESA, p. 71.

Ryutova M., Kaisig M., Tajima, T., 1992, in preparation. 